\title{
COMPARATIVE STUDY OF LITHIUM ION HYBRID SUPER CAPACITORS
}

\author{
Leslie R. Adrian ${ }^{1,2}$, Donato Repole ${ }^{1}$, Aivars Rubenis ${ }^{3}$ \\ ${ }^{1}$ Riga Technical University, Latvia; ${ }^{2}$ SIA “Lesla Latvia”, Latvia; \\ ${ }^{3}$ Latvia University of Life Sciences and Technologies, Latvia \\ leslie.adrian@rtu.lv, donato.repole@rtu.lv, aivars.rubenis@transfoelectric.com
}

\begin{abstract}
A relative newcomer to the energy storage market, the Lithium Ion Hybrid Super Capacitor is a novel technology breaking new ground in the technology sector. The (LIC) or (LIHC) is fast evolving as the missing link between the Electric Double Layer Capacitor (EDLC) and the Lithium Ion Battery (LIB), being a distinct hybrid of the two technologies. The LIHC combines both energy and power with far longer life and safety features. The use of LIHC capacitors has already woven itself into many industry applications including but not limited to hybrid vehicles, remote area charging solutions, energy harvesting and storage and communications technologies. Mass deployment of LIHCs seems inevitable given the uptake in design processes with the pros outweighing the cons and with higher volume production increasing rapidly. In this paper we will model the Lithium Ion Capacitor characteristics and explore how they perform against an equivalent rival, the standard EDLCwith specific focus on the instantaneous initial charge performance of Lithium Ion Capacitors compared to the other. The focus of this study model is the behaviour of a standard EDLC Super-capacitors Equivalent Series Resistance, "ESR" versus an LIHC Super-capacitor "ESR" of comparable specification in the initial seconds of state of charge.
\end{abstract}

Keywords: lithium, ion, hybrid, super capacitor.

\section{Introduction}

Lithium Ion Batteries are currently the predominant choice in applications requiring mobile or power storage requirements, due to their high energy density, however their low power density and inadequate cycling life in many instances impede their potential for performance.

Not too unlike the LIBs the EDLCs, despite having a significant role to play in various applications, are endowed with very high power density but sadly lacking in energy density [1], where if today the Lithium Ion Batteries (LIB) are dominant in these applications due to their high energy density, their lack of power density and their limited cycle life sometimes hinder the performances of the system in which they are used. Super-capacitors, known as Electric Double Layer Capacitors have a very high power density but are limited in terms of energy density. So, as a matter of choice the LIBs were an outright winner, even though the cycling life of the EDLC is many orders of magnitude greater than the LIB. Nowadays more importance is being placed on energy harvesting, energy storage and alternative energy sources than ever before and the Lithium Ion Super Capacitor has emerged in recent years as a fill gap between LIBs and EDLCs having features which are intermediary to both LIBs and EDLCs.Due to the overlying hybrid architecture, an LIHC can operate between $2.2 \mathrm{~V}$ and $3.8 \mathrm{~V}$, which represents a comparable voltage range to LIB.LIHC cells may be discharged and charged at high currents, with their voltage displaying a near linear relationship to State-of-Charge. This simplifies monitoring in comparison to LIB, which require for the most part a charge protocol, which is not required for the LIHC.

Having a much lower energy density than LIBs, super-capacitors have traditionally been classed as too expensive in Euro/kWh, however their energy storage per $\mathrm{kWh}$ requirement is considerably less than LIBs in power applications due in the main to the much lower power capability of the LIBs in $\mathrm{kW} / \mathrm{kg}$, which in turn effects the energy density of the storage unit [2]. For portable and mobile devices LIBs are considered to be an ideal technology due to their extremely good energy density, however in the instance of and requirements of regenerative braking to improve both deceleration and acceleration in all types of electric vehicles, the sole or combined use of LIBs or EDLC supercapacitors fall short in their ability to provide instantaneous response in the initial seconds of charge [3], where it is clearly described that to improve the acceleration and deceleration performance of electric vehicles, an application of hybrid energy storage consisting of batteries and super-capacitors or batteries and lithium capacitors is indispensable. It therefore follows that an LIHC, which in effect is a hybrid between LIBs and EDLCs, could form a future solution reliant only on continued improvement of the current energy and power densities. 
It is unfortunate that little comparison may be found to further consolidate the model findings within this paper due to the heavy reliance placed on the reporting of thermal characteristics, product longevity, cycling and energy and power densities of these devices which are far more prevalent in the literature. In the area of instantaneous voltage and current in the first moments of charge these parameters are of critical importance for the improvement of acceleration and deceleration methods of regeneration used in electric vehicles.

\section{Model Design Assumptions}

An Electric Double Layer Capacitor (EDLC),(AVX SCCY85B607SLBLE 600F) and aLithium Ion Hybrid Super Capacitor (LIHC Lesla-Technologies/CDA) 750Fwere selected for the study case with relevant specifications as in Table 1. Calculation of Accumulator banks may be seen (1) and (2).

EDLC Bank: 22s x 2p where each unit uses a volume of $79.86 \mathrm{~cm}^{3}$ for an accumulator capacitance of $\sim 54 \mathrm{~F}$.

$$
\text { Volume }_{E D C L}=22 \cdot 2 \cdot 79.86 \mathrm{~cm}^{3}
$$

LIHC Bank: 15s x 1p where each uses a volume of $10.18 \mathrm{~cm}^{3}$ for an accumulatorcapacitance of $\sim 50 \mathrm{~F}$.

$$
\text { Volume }_{\text {LIHC }}=15 \cdot 10.18=152.7 \mathrm{~cm}^{3}
$$

- EDLC: $0.0154 \mathrm{~F}$ for each $\mathrm{cm}^{3}$.

- LIHC: $0.33 \mathrm{~F}$ for each $\mathrm{cm}^{3}$.

This equates to 21.4 times the capacitance-volume ratio compared to the EDLC Capacitor used as the baseline, or in percentage terms $4.35 \%$ of the EDLC.

\begin{tabular}{|c|c|c|}
\hline Type & EDLC & LIHC \\
\hline Diameter, mm & 35 & 18 \\
\hline Length, mm & 83 & 40 \\
\hline Rated capacitance, F & 600 & 750 \\
\hline Rated Voltage (min/max), V & $2.3 / 2.7$ & $2.5 / 3.8$ \\
\hline ESR Max @ 1000 Hz, m $\Omega$ & 1.6 & $\leq 25$ \\
\hline ESR Max @ DC, m $\Omega$ & 1.8 & $\leq 26$ \\
\hline Peak Current, A & 389.42 & $*$ \\
\hline Power Density, W· $\mathrm{kg}^{-1}$ & 4459 & $*$ \\
\hline Max Energy, Wh & 0.6075 & $*$ \\
\hline Energy Density, Wh$\cdot \mathrm{kg}^{-1}$ & 5.57 & $*$ \\
\hline
\end{tabular}

Selected test types

The undeniable decrease in the capacitance-volume ratio is further accentuated by the Depth of Discharge (DOD) problems associated with LIBs and to recognize evaluations which must be undertaken in order to determine their true Power and Energy Density when compared to the LIHC devices [5], which are capable of full chargeand discharge over an exceedingly long life cycle.

\section{Model Simulation Experiment Verification}

In order to verify the validity of the designed strategy in this paper, a simulation model has been built in LTSpice in accordance with information extracted from both capacitor type data sheets for verification as in Fig. 1 and Fig. 2. The system parameters are shown as indicated in Table 2 below.

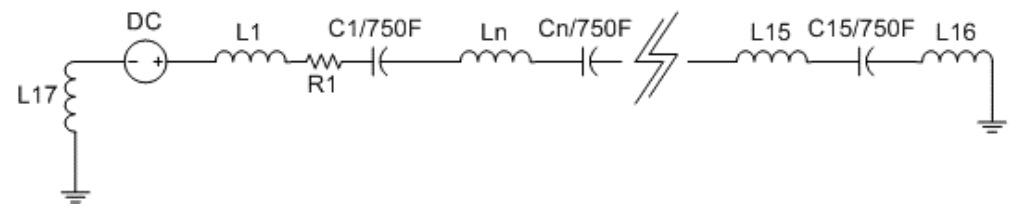

Fig. 1. Charging simulation schema for the LIHC Super-capacitor $48 \mathrm{~V}$ Bank 


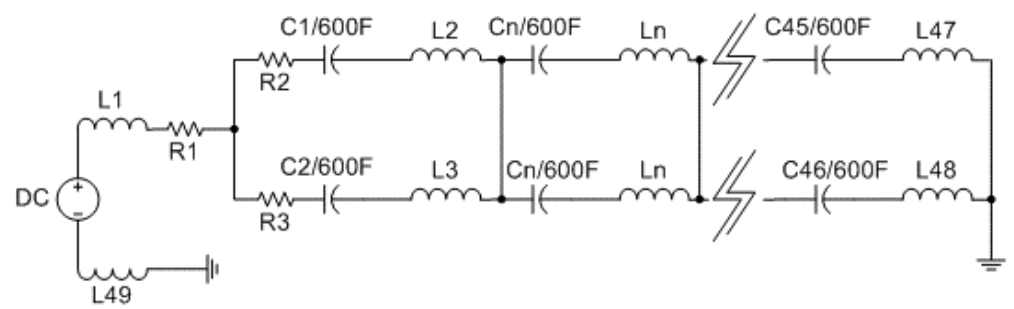

Fig. 2. Charging simulation schema for the EDLC Super-capacitor 48V Bank

Table 2

Simulation parameters

\begin{tabular}{|c|c|}
\hline Parameter & Value \\
\hline DC Regulated Voltage & $48 \mathrm{~V}$ nominal - Overvoltage tip 53.2 \\
\hline Max Peak Current & $160 \mathrm{~A}$ \\
\hline Charger continuous Power Rating & $2 \mathrm{~kW}$ \\
\hline Super capacitor (EDLC) & $54 \mathrm{~F}$ \\
\hline Super capacitor (LIHC) & $50 \mathrm{~F}$ \\
\hline Element to PCB - Stray Inductance & $2 \mathrm{nH}$ \\
\hline $\begin{array}{c}\text { Estimated Overall Stray Inductance } \\
\text { (excluded connection cables) }\end{array}$ & Approx. $100 \mathrm{nH}$ \\
\hline
\end{tabular}

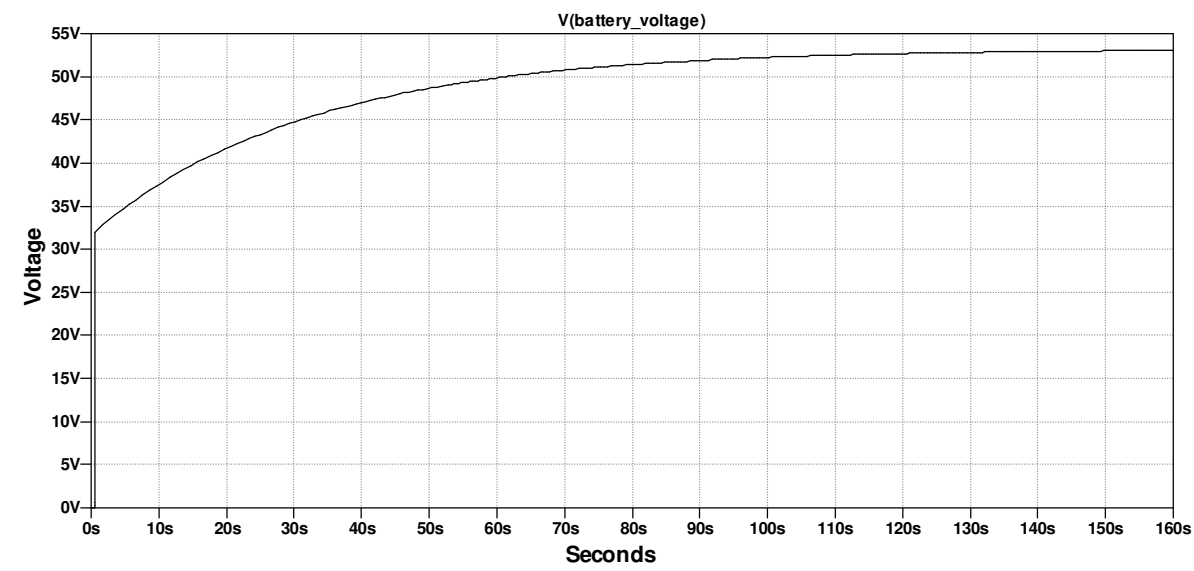

Fig. 3. EDLC 48V Bank: battery voltage charging simulation

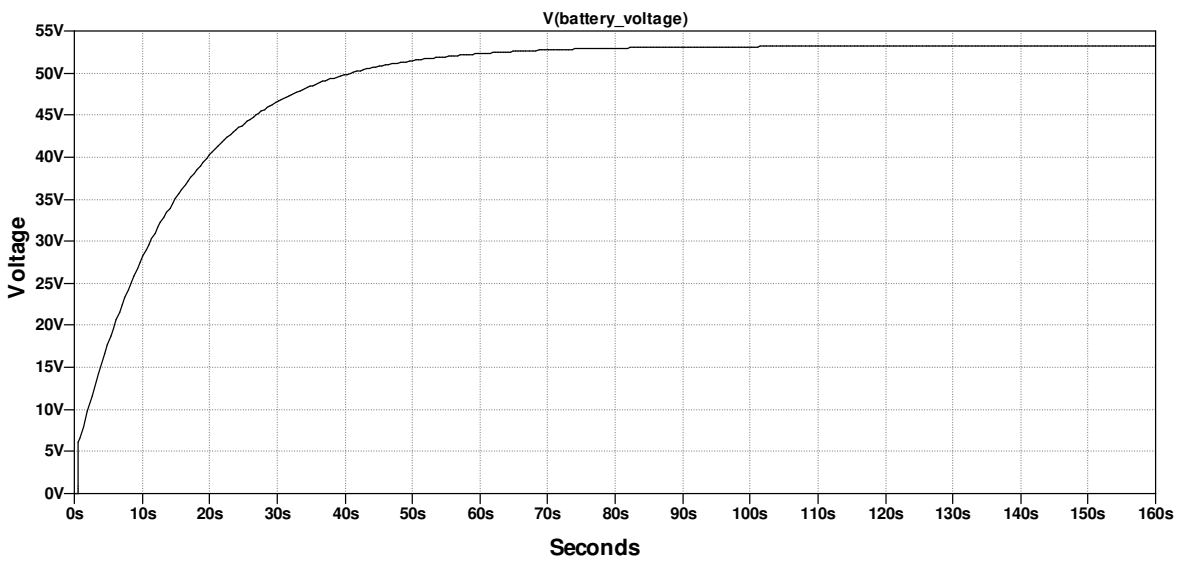

Fig. 4. Lithium Ion Capacitor 48V Bank: accumulator battery voltage charging simulation 
The LIHC is able to reach steady state voltage more rapidly than the EDLC, as the operative voltage that should be applied to the accumulators is approximately $3.55 \mathrm{~V}$ for the LIHC and $2.3 \mathrm{~V}$ for the EDLC, respectively. See Fig. 5 and Fig. 6.

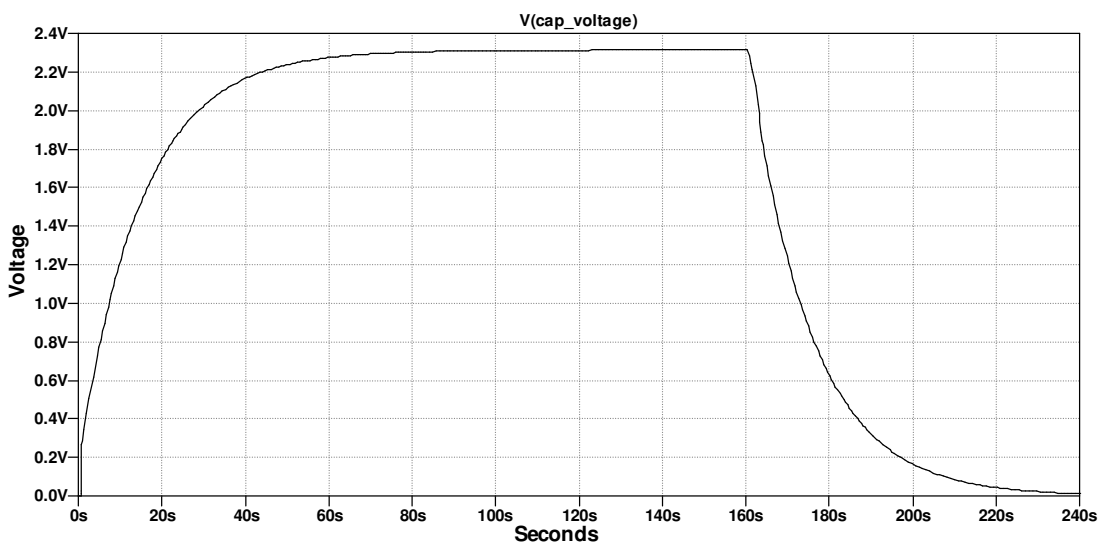

Fig. 5. Steady state voltage of EDLC capacitor bank

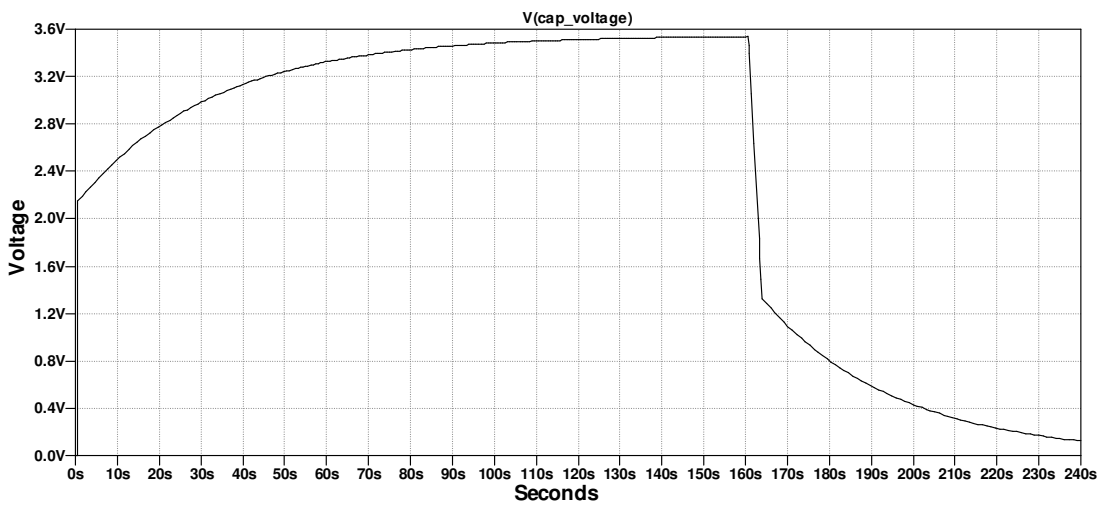

Fig. 6. Steady state voltage of LIHC

\section{Investigation and Bench Test Preparation}

The investigation targets two study cases:

- regenerative braking;

- battery fast charging.

The first simulation targets the behavior of a $48 \mathrm{~V}$ (nominally rated, with overvoltage tip of $53.2 \mathrm{~V}$ ) Super-capacitor accumulator unit during a full charge achieved by regenerative braking. This research does not study the end application and the regenerative system, but focuses exclusively on the comparison between the LIHC technology and an off the shelf EDLC technology in the initial charge state.

The second investigation is proposed to deliver a full data spectrum for a single cell charge, which may be associated to any fast charger application, with the primary goal of comparing the physical results with the output data of the simulations and thereafter proceed to the validation of the simulation conclusions.

According to earlier description, the simulation considers two accumulators having similar characteristic, although they are not identical. The accumulator simulations highlight that the electrical characteristics of the AVX-EDLC Technology are capable of operating with a higher peak current and higher charging currents, although the LIHC Technology shows a smoother charging profile. This indicates that when the accumulator is wholly discharged the AVX-EDLC accepts higher charging current and accumulates energy more rapidly (with its higher dv/dt) than the LIHC technology. As soon as the accumulator voltage increases and is closer to the accumulator nominal voltage, the 
accumulator in the LIHC technology will charge quickly (higher $d v / d t$ ) due to the fact that the accumulator in the LIHC Technology is composed of fewer elements to attain an equivalent capacitive-volume than the EDLC bank. Looking at the time required to reach the nominal accumulator voltage, the LIHC technology indicates the capability to be charged more rapidly.

Confirmation is required by a physical test, which should indicate the supremacy of the LIHC technology over the AVX-EDLC technology.

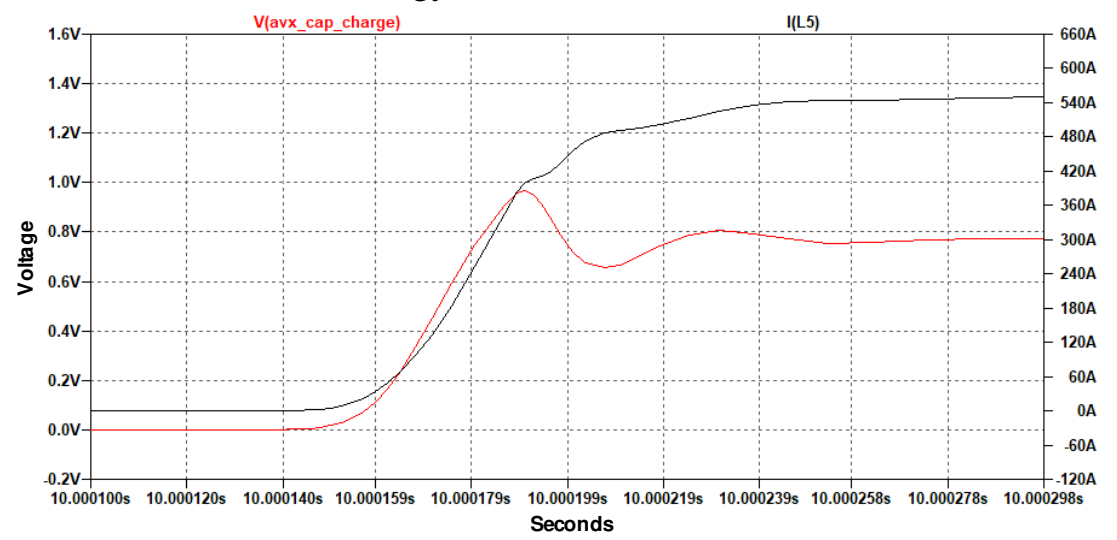

Fig. 7. EDLC Inrush current/voltage behaviour in front of fast energy storage discharge

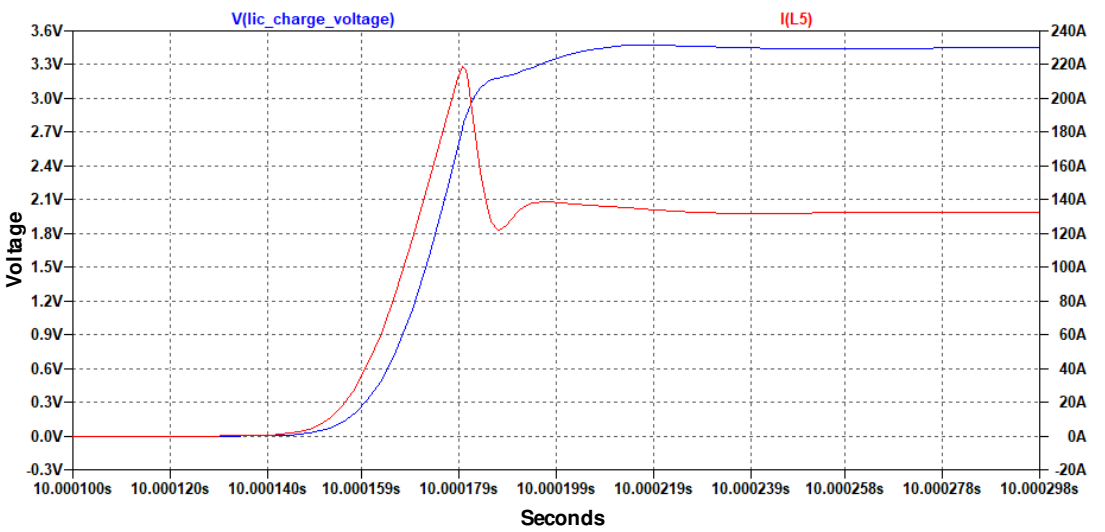

Fig. 8. LIHC Inrush current-voltage behaviour in front of fast energy storage discharge

The test bench is to be constructed with the following devices:

- 1 x 200MHz laboratory oscilloscope

- 1 x 3A capable power supply

- 1 x Signal generator (control of mosfet)

- 2 x ST STH300NH02L-6 mosfets

- 5 x LIHC Super-capacitors

- 6 x EDLC AVX Super-capacitors

The peak current that should be provided during the first phase of the charge is very high, rendering it unfeasible to use a powerful supply. To build a cost effective test-rig, a simple power supply which will charge 3A constant current, a hybrid set of EDLC and LIHC super-capacitors, as Fig. 9 and 10 below, for a total capacitance of 2850F (and will continue to feed up 3A to the bulk capacitor once it will be discharged) and transfer part of the charge to the testing capacitor.

The hybrid capacitor charged at the most appropriate voltage during its discharging operation charges the test capacitor, the physical switch is implemented with 2 x STH300NH02L-6 (180A DC and 720 pulse current for a combination of $360 \mathrm{~A}$ and $1.44 \mathrm{kA}$ pulse current rating). 


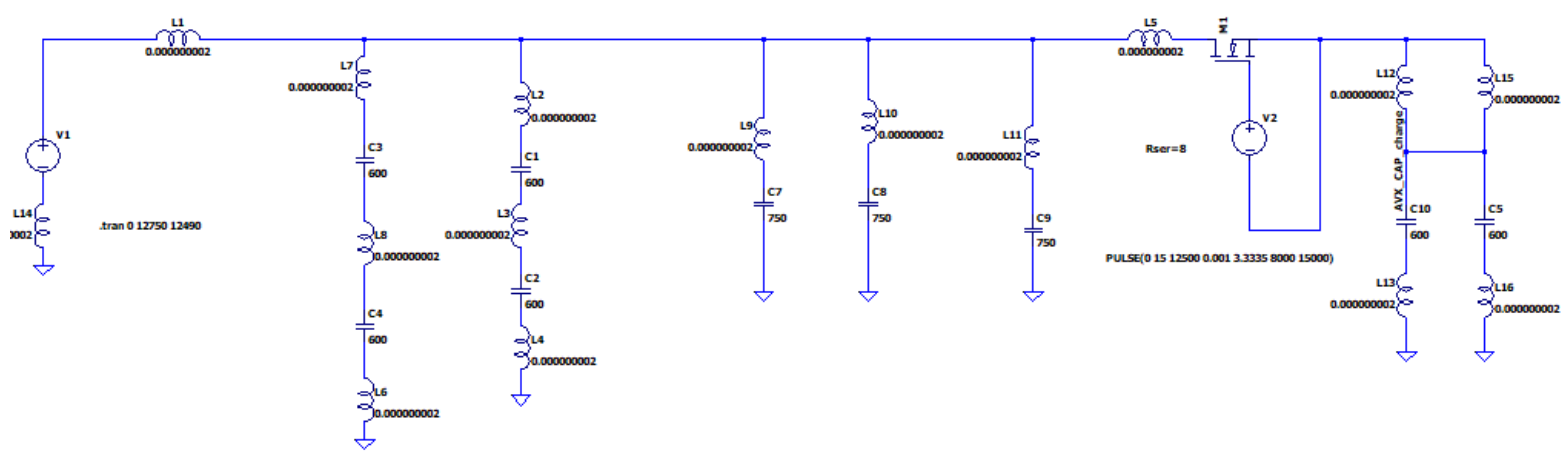

Fig. 9. Bench test schema for AVX EDLC

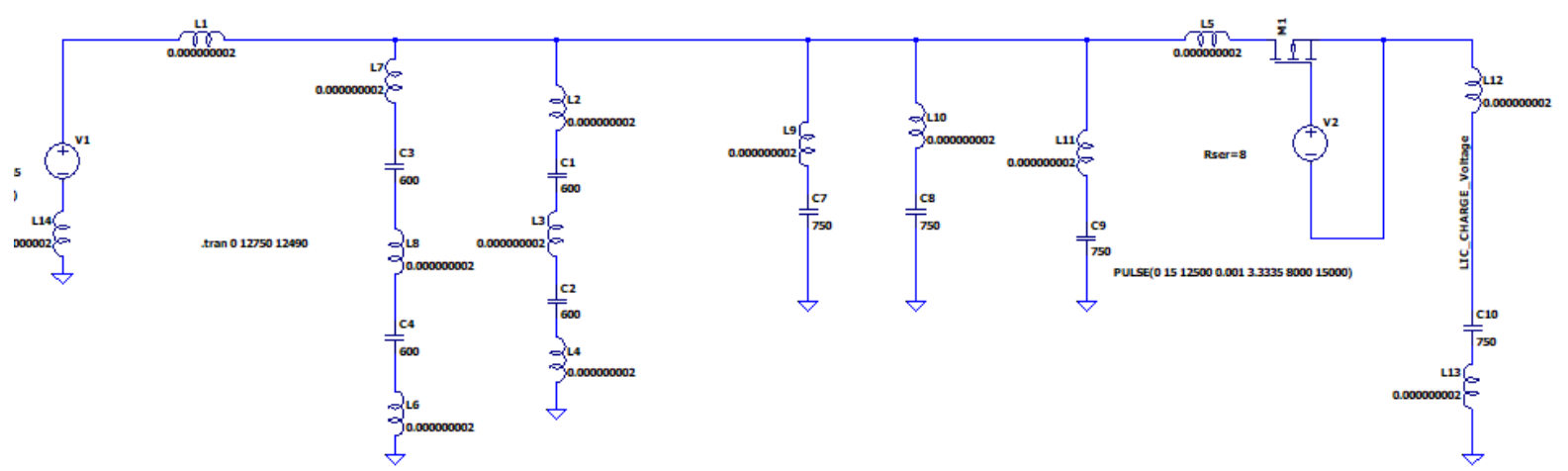

Fig. 10. Bench test schema for Lesla Technologies LIHC

The above bench test schema is not strictly necessary for this initial stage, as the results of simulation closely align with Figures received from factory testing documents acquired during the course of the investigation. However, for the purpose of completeness have been included and will be necessary in future power and energy density research.

\section{Results and discussion}

It is important to highlight important comparative findings from the simulations:

- The LIHC super-capacitors are much more space effective than the EDLC for the construction of energy accumulators rated to a $48 \mathrm{~V}$ battery.

- LIC super-capacitor reaches the steady state voltage more rapidly than the EDLC.

- The EDLC have the benefit of having a far lower ESR, which is attenuated by the necessity to use more devices in series and in parallel, which increments the current peak necessary to charge the device resulting in a slower charging graph. (This allows a longer discharge of the capacitor, however this is not beneficial for this study case).

- The high power capability in a compact size, which allows to have a higher energy content inside the same volume compared to an EDLC solution.

- The life cycle of the device makes it needless in many applications to replace during the life of the product.

- The higher robustness of the LIC at higher temperatures, which can reduce cooling requirements.

\section{Conclusions}

In this paper a simulation analysis has been performed of a LIHC $48 \mathrm{~V}$ accumulator model in comparison with the equivalent model of a EDLC $48 \mathrm{~V}$ accumulator. In order to have an accurate model, specifications and associated guidelines for factory testing have been followed which has evolved into similar results being verified. The initial charge status has indicated that although both models show a charge initiation at $T=640 \mathrm{mS}$, the EDLC Bank receives charge far more rapidly and 
instantaneously to approximately $32.5 \mathrm{~V}$, thereafter proceeding to much slower than the LIHC steady state. The LIHC reaches almost instantaneously the voltage of $7 \mathrm{~V}$, thereafter is proceeding to steady state. Looking at the rise time necessary to reach the nominal voltage (48V) quicker than the EDCL. This outcome appears to correspond with an implied need to examine the use of LIHCs over EDLCs for both regenerative charging and fast battery charging. The results indicate that the above included with an examination of matters regarding power and energy density, as described earlier, should be the subject of future research.

\section{Acknowledgements}

This work has been supported by the European Regional Development Fund within the Project "Development of a Sustainable Heating Solution for the Salon of the Public Electric Transport", project identification number 1.1.1.1/18/A/168

\section{References}

[1] Ronsmans J., Lalande B. "Combining energy with power: Lithium-Ion Capacitors," 2016 International Symposium on Power Electronics, Electrical Drives, Automation and Motion (SPEEDAM), Anacapri, 2016, pp. 261-264, DOI: 10.1109/SPEEDAM.2016.7525905.

[2] Burke A., Zhao H., "Present and Future Applications of Supercapacitors in Electric and Hybrid Vehicles," 2015 IEEE 82nd Vehicular Technology Conference (VTC2015-Fall), Boston, MA, 2015, pp. 1-5, DOI: 10.1109/VTCFall.2015.7391093.

[3] Soltani M., Ronsmans J., Jaguemont J., Mierlo J. V., Van den Bossche P., Omar N. "A Threedimensional thermal model for a commercial lithium-ion capacitor battery pack with non-uniform temperature distribution,” 2019 IEEE International Conference on Industrial Technology (ICIT), Melbourne, Australia, 2019, pp. 1126-1131, DOI: 10.1109/ICIT.2019.8755081.

[4] Yunhong Z., Aimin Z. Hang Z. "A Super Capacitor Charging Device Based on the Sliding Mode Control Strategy," 2018 Chinese Automation Congress (CAC), Xi' an, China, 2018, pp. 30143018, DOI: 10.1109/CAC.2018.8623305.

[5] Xu B., Oudalov A., Ulbig A., Andersson G., Kirschen D. Modeling of Lithium-Ion Battery Degradation for Cell Life Assessment. IEEE Transactions on Smart Grid. 99. 1-1. 2016. DOI: 10.1109/TSG.2016.2578950. 